\title{
Ecological Interface Design for Teaching Assembly Operations to Industrial Robot *
}

\author{
Yukio Horiguchi* Kohei Kurono* Hiroaki Nakanishi* \\ Tetsuo Sawaragi ${ }^{*}$ Tatsuya Nagatani ${ }^{* *}$ Akio Noda** \\ Ken'ichi Tanaka** \\ * Department of Mechanical Engineering and Science, Kyoto \\ University, Yoshida Honmachi, Sakyo-ku, Kyoto 606-8501 Japan \\ (e-mail: horiguchi@me.kyoto-u.ac.jp). \\ ** Mitsubishi Electric Corporation, Japan
}

\begin{abstract}
This study analyzes the work domain of position teaching in terms of means-end relations for the purpose of developing a practical support tool for human workers engaged in industrial robot teaching. A mechanical explanation model is introduced into the analysis to capture the force-displacement relationship inherent in and informative on the work system. The resulting models are used for the analysis of the robot operators' decisions in search of accurate operation positions, and this in turn helps to clarify a rational operation strategy for making use of an invariant frame of reference in the position search space. After these findings, a prototype GUI is proposed that can provide effective information supports for different granularities of the activity in position teaching. Based on the principles of Ecological Interface Design, the proposed GUI represents the activity-related information in a way that encourages the robot operators' intuitive and strategic operations.
\end{abstract}

Keywords: Ecological interface design, human-machine interface, industrial robots, robot teaching, work analysis.

\section{INTRODUCTION}

Almost all conventional methods for teaching assembly motions to industrial robots depend on the teachingplayback system which requires fitting each operation position of the robot to the actual spots where that robot should work. The more complex operations like parts mating are, the more position data must be taught to the robot. A sufficient amount of accuracy of positioning is another requirement for realizing the robot's finer automatic error-free operations. Position teaching is therefore one of the most demanding and time-consuming tasks, but few studies have been conducted to investigate robot teaching tasks in practice and to develop a practical level of support for human workers. This research aims at developing effective information supports to assist the workers engaged in such a hard task and to streamline the robot teaching works.

Redesigning the human-machine interaction involves a deeper understanding of the constraints underlying the work system as well as of the cognitive strategies to make the most use of the available information and to cope with the difficulties in the current interaction design. These are valuable sources for extracting the semantic structure embedded in the work domain, and this structure is to be

* This research has been partially supported by the Intelligent Robot Technology Software Project of New Energy and Industrial Technology Development Organization (NEDO) of Japan, and by a Grantin-Aid for Creative Scientific Research 2007-2011 (No.19GS0208) of the Ministry of Education, Culture, Sports, Science and Technology (MEXT) of Japan. We are grateful for their support. visualized in the external resources directly available to human users. In order to define the information requirements of the robot teaching works, this study analyzes the work domain of "position teaching" in terms of means-end relations, and then introduces a mechanical explanation model to the resultant work domain model in order to capture an important aspect of the causal relationship inherent in the work system. This model is used for the analysis of the worker's operation strategy in search of accurate operation positions. The series of analyses used in this study aim at clarifying the invariant features that can be used to characterize the rationale for steadily and efficiently conducting position teaching.

After our findings from these analyses, a prototype GUI is proposed that can provide effective information supports for the different granularities of activity in position teaching. Based on the principles of "Ecological Interface Design" (Vicente and Rasmussen (1990); Vicente (2002); Burns and Hajdukiewicz (2004)), the proposed GUI consists of configural displays used to represent the activityrelated information in a way that encourages the operators' intuitive and strategic operations.

\section{ANALYSIS OF ROBOT TEACHING WORK}

\subsection{Target Work Domain}

Generally speaking, the following procedural steps are used for creating programs for automatic robot operations (e.g., Mitsubishi Electric Corporation (2009)). 


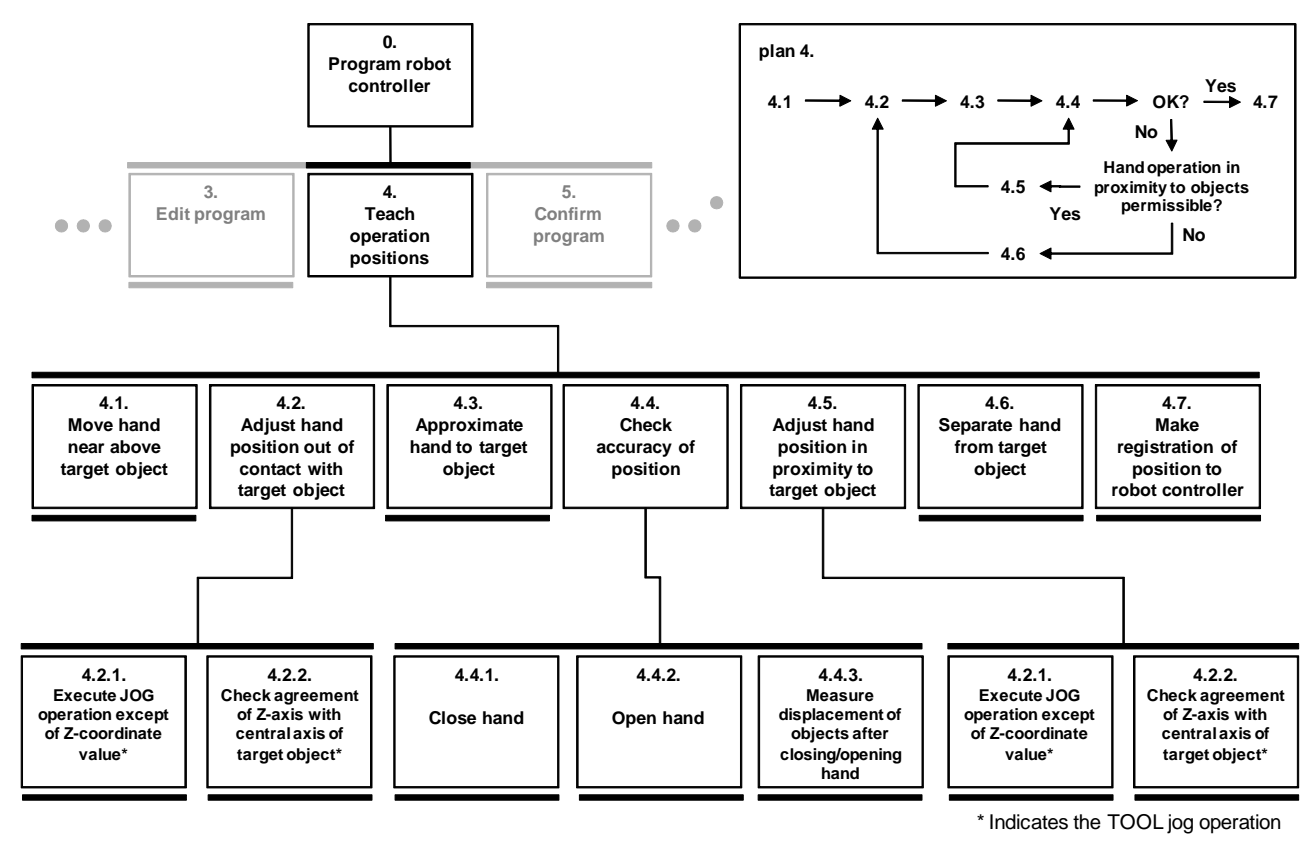

Fig. 1. Hierarchy of robot programming task with special focus placed on position teaching operations

1. Decide on the robot operation order, operation path, and work at each operation position such as opening and closing hand.

2. Define the position variables to be taught for generating the intended operation path.

3. Based on the decided operation order and position variables, convert the robot operations and works into commands to create the robot program.

4. Manually move the robot to each operation position (jog operation), and teach them the position variables.

5. Execute the program line by line, and confirm that the program and operation positions are correctly taught.

6. If any mistakes are found in the robot operations or works during the program confirmation, go back to step 3. to correct the program and/or operation positions.

Position teaching (step 4.) is the most time-consuming and demanding task among these steps because accurately determining the positions for the automatic robot operations in an actual task environment is a continuing process of trial and error. The more complex the operations the robot should perform, the more position data are required to be taught. In this phase, a lot of communication will therefore appear between the human operator and the robot system. The operator support through the use of information displays is promising for improving the overall work efficiency.

The task of position teaching was further broken into subtasks by using the Hierarchical Task Analysis technique (Shepherd (2000)). Fig. 1 shows the results of the analysis, illustrating that the reputation of the adjustments and checking of the robot positions is necessary for achieving accurate target positions. These works conventionally depend fully on the visual observation of the physical interaction between the robot hand and the target object. Subtle displacements or deformations of the objects after closing or opening the hand are only a single source of information for evaluating the accuracy of the current hand position. Information enrichment is especially required for human operators at this stage of work. That is why we selected the technical system related to the tasks 4.4 and 4.5 as the target domain of the analysis.

\subsection{Analysis of the Work Domain}

Work Domain Analysis Work Domain Analysis (WDA) was applied to the robot system in order to examine what kind of information should be specified for supporting quick and correct decision in position teaching. WDA identifies the means-end relationships between the system's functions at different levels of abstraction. A means-end relation reveals the functions at one level that must be used for satisfying a function at a higher level. Abstraction Hierarchy, which is the product of WDA, represents the functional structure of a system organized from the functional purposes to the physical components of the system (Rasmussen (1986); Vicente (1999)).

Fig. 2 shows part of the abstraction hierarchy of a work domain whose purpose is to find the most accurate operation position to be taught to the robot. This model illustrates that the checking and adjustment of a robot's position are implemented by means of a hand positioning process, and that a causal relationship mediates the connections between the purpose ("accuracy of operation position") and the functional properties such as the ("hand positioning") process. This relationship involves the strain energy stored in the objects because any disagreement between the hand position and the fixation position of the target object will generate some deformation in their interlinked structure when the hand is grasping that object. Although the energy in the structure holds a lot of information about how to resolve the positional and postural errors of the hand, nothing about it is explicitly provided to the operators using more conventional ways of position 


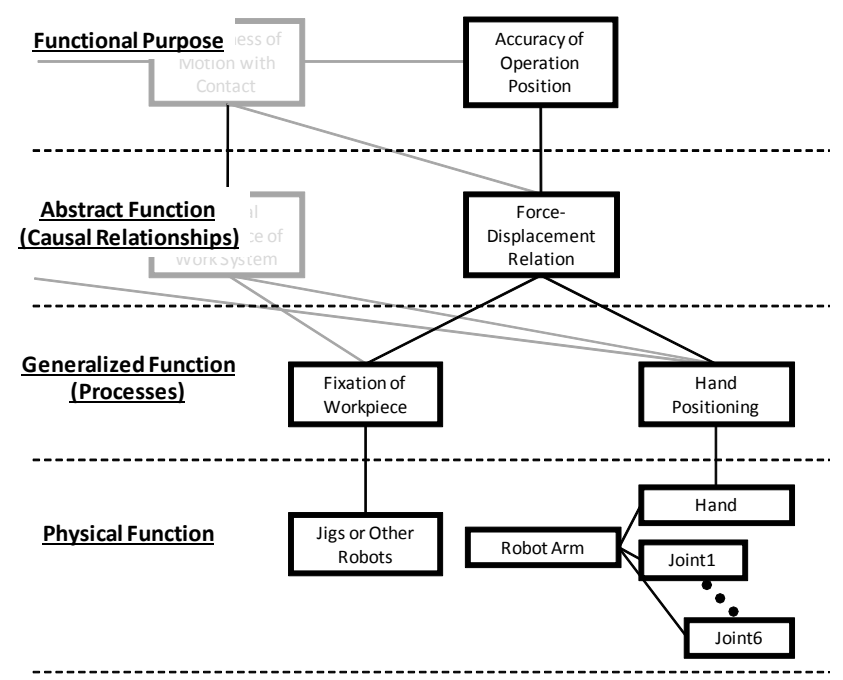

Fig. 2. Abstraction hierarchy of functions related to achievement of accurate operation position
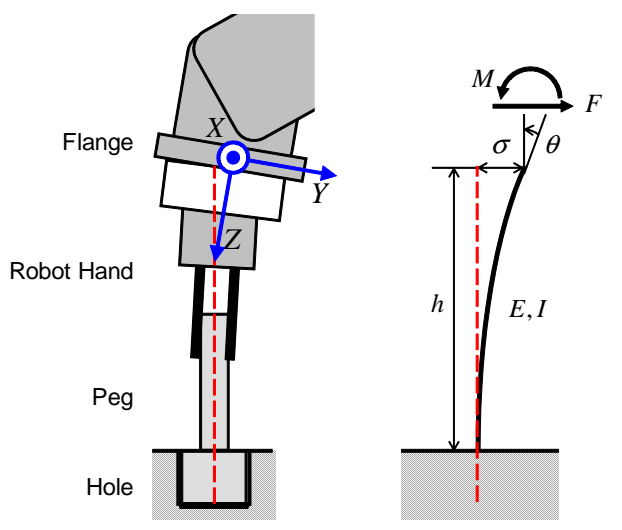

Fig. 3. Modeling state of robot hand grasping an object using standing cantilever beam

teaching. They have to speculate the system state from the object's motion by opening and closing the hand (task 4.4) over and over. This may require a lot more trial and error time to the human operators.

Modeling the Causal Relationship We used a freestanding cantilever beam to more accurately model the causal relationship related to position teaching. As shown in Fig. 3, this mechanical model portrays a state in which the robot hand is grasping a peg inserted into its corresponding hole. The purpose of this work system is to define the optimal position of the hand in order to grasp the peg. The horizontal position error and posture error of the center of the flange face relative to the central axis of the hole are used to quantify the degree of task achievement. The coordinate axes drawn in the figure represent a tool coordinate system (Mitsubishi Electric Corporation (2009)) with no tool length, which is usually used for the subtle positioning of the robot hand in position teaching.

For the sake of simplicity, only two dimensions of the system state are considered here as depicted in Fig. 3. $\sigma$ and $\theta$ signify the horizontal position error and posture error of the system. $F$ and $M$ represent the force and moment that are applied to the free end. The model assumes that there is no displacement between the peg and the hole, and that $\theta$ is so small that the inclination of the coordinate axes can be ignored.

Based on the differential equation of the deflection of the beam, the $\sigma$ and $\theta$ errors can be described in terms of $F$ and $M$ as follows.

$$
\sigma=\frac{1}{E I}\left(\frac{1}{3} F h^{3}-\frac{1}{2} M h^{2}\right),
$$

and

$$
\theta=\frac{1}{E I}\left(-\frac{1}{2} F h^{2}-M h\right),
$$

where $E$ and $I$ represent the Young's modulus and the geometrical moment of inertia, respectively. From (1) and (2), we derive

$$
F=12 E I \frac{\sigma}{h^{3}}+6 E I \frac{\theta}{h^{2}}
$$

and

$$
M=6 E I \frac{\sigma}{h^{2}}+4 E I \frac{\theta}{h} .
$$

Equation (3) determines that $F(\sigma, \theta)$ forms a flat surface that passes through the origin in a three-dimensional space whose components are $\sigma, \theta$, and $F$. The same goes for (4) about $M(\sigma, \theta)$.

As shown in the above equations, the forces within a structure can provide more specific information on how to resolve the errors than the conventional ways can. However, they are interrelated as described in (3) and (4), and their relations involve constants, such as $E$ and $I$, that are hard to identify within a work. So, the force measurements underspecify the actions for improving the situation, and some strategic operations are required to eliminate them all together.

\section{STRATEGY TO USE FORCE INFORMATION}

The processes used in searching for accurate operation positions are analyzed based on the relations derived from the cantilever model, in order to determine the utilization strategies of the force and moment information in position teaching.

\subsection{Experiment}

Experimental Setup An experiment was conducted to collect all the necessary operational data when the information concerning the forces being exerted on a robot was available to human operators. The platform for the experiment was developed using the Mitsubishi Electric Industrial Robot RV-6SL and Nitta's 6 DoF force/torque sensor IFS-67M. The sensor was installed onto the robot's wrist, which was used to measure the forces and moments at the center of the flange face.

We used a cylinder-shaped peg made of stainless steel as our target object, and it was inserted into a circular hole with a brass contacting surface. The diametral gap between the peg and the hole was $10 \mu \mathrm{m}$. The participant 


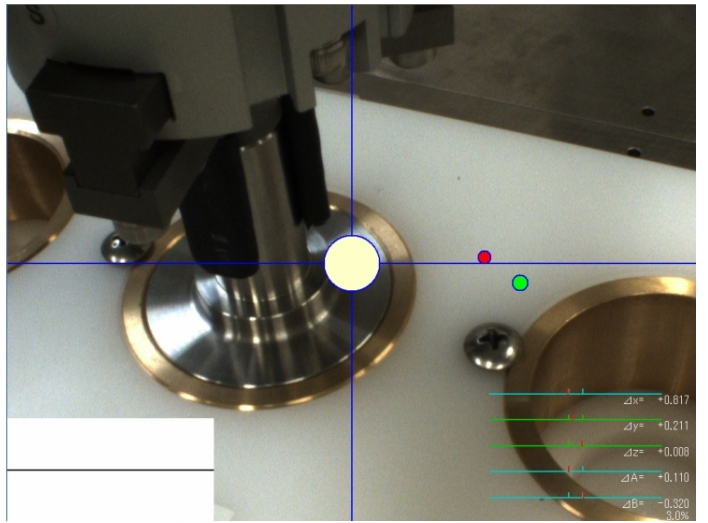

Fig. 4. Information display visualizing forces and moments exerted on robot's wrist

operators tried to determine the operation position at which the robot would be able to insert the peg into the hole. The experimenter arbitrarily set the initial position of the robot for each work session, and no information about it was provided to the participant operators. The operations for moving the robot were commanded by means of a "teaching pendant" that was actually a remote control device used for jog operations. The jog operation mode was set to the LOW mode in which the minimum distance of translation and the minimum angle of rotation were $0.01 \mathrm{~mm}$ and $0.1 \mathrm{deg}$, respectively.

The measurements taken by the force/torque sensor were presented on a two-dimensional surface display. As shown in Fig. 4, the live camera image capturing the area around the robot hand for vigilance forms the background of this display. The red point represents the pair of force measurements $\left(F_{X}, F_{Y}\right)$, while the green point represents the pair of moments $\left(M_{Y} / l, M_{X} / l\right)$ where $l$ was a constant to fit their unit to the forces. The white circle at the center of the display represents the target region into which both the pointers should be moved by positioning the hand. A pointer inside the circle denotes that the forces or moments have been reduced to less than their criterion of $1 \mathrm{~N}$ or $0.15 \mathrm{Nm}$, and if both pointers are inside the region at the same time it represents the completion of the task.

Result Fig. 5 presents a summary of the experimental results, in which "FID" stands for the work condition with a force information display, whereas "No FID" refers to the one without it. The values in the former condition are the averages from nine sessions performed by three novice operators, to each of whom three sessions were assigned in the experiment. In the latter condition, a middle-level operator conducted six work sessions without reference to the force information (i.e., in a conventional way of position teaching). These results confirm that the information display allows the operators to more efficiently conduct position teaching although they had had no experience in robot teaching. In terms of both the task time and position accuracy (evaluated by the norm of forces when the teaching task had been finished), the novices outperformed the middle-level operator, and their performances had fewer variations.

On the other hand, several of the operations in search of adequate positions involved missteps despite the force

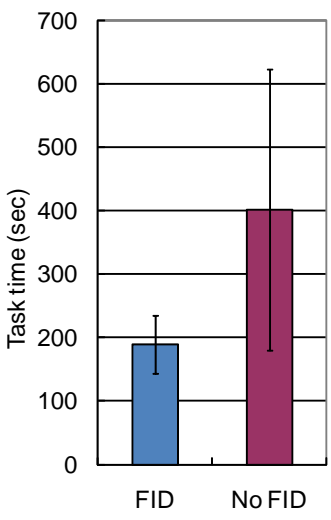

(a) Task time

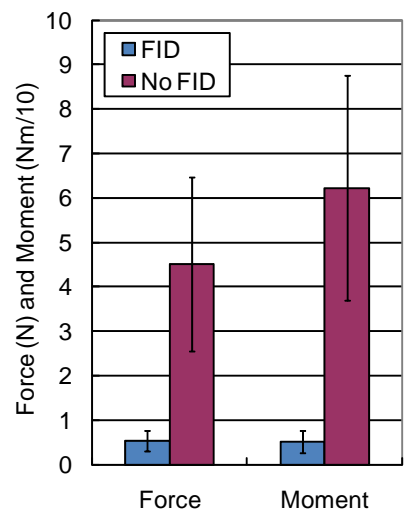

(b) Position accuracy
Fig. 5. Comparison of teaching performance between with and without force information display

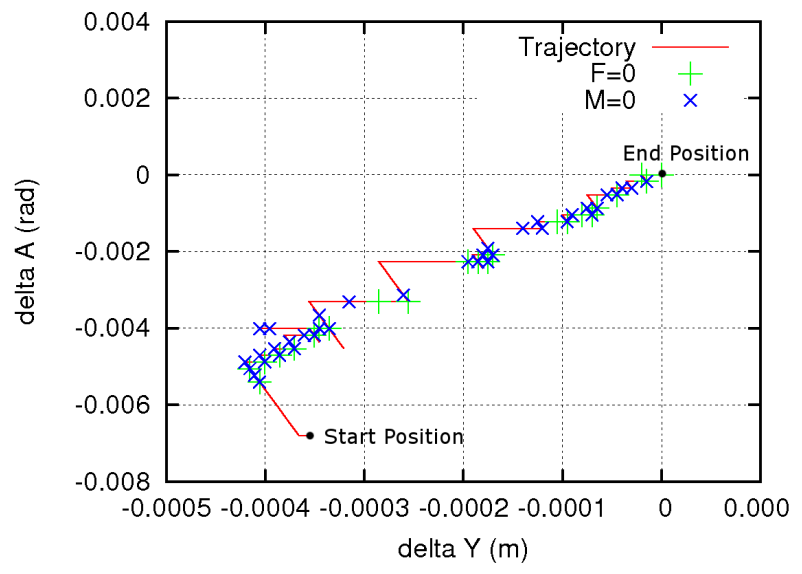

Fig. 6. State trajectory in search of an accurate operation position

information being made available. Referring back to (3) and (4), these relations do not exactly specify which and how much translation or rotation should be applied to the robot hand. This force information underspecifies the actions for improving the situation. So, some explorations are needed to determine the correct search direction, and the search strategy should be firmly maintained even after the correct direction has been identified. This issue is discussed in particular in the next subsection.

\subsection{Processes in Search of Accurate Operation Position}

Similarly to the information and situation outlined in the previous section, the analysis was confined two dimensionally: Only the translation along the Y-axis and the rotation around the $\mathrm{X}$-axis - 'Y-axis operation' and 'A-axis operation' - are considered in the tool coordinate system whose origin is located at the center of the flange face (Fig. 3).

Fig. 6 shows a operational case for the FID condition as a trajectory on the Y-A surface. In this state space, the completion of the task (i.e., the state with no positional and postural errors) corresponds to the coordinate origin 


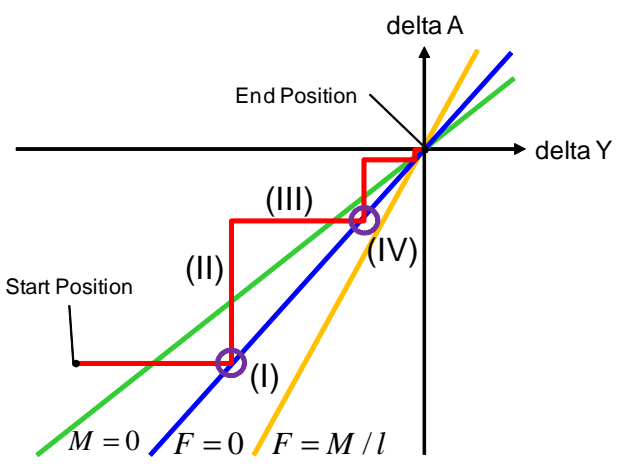

Fig. 7. Diagrammatic illustration of successful trajectory in search of accurate operation position

$(0,0)$, and the lateral movements represent the Y-axis operations while the longitudinal movements represents the A-axis operations. Strictly speaking, an A-axis operation generates the longitudinal movement as well as the lateral one because the rotation around the center of the flange face simultaneously makes a translational movement in the grasp position. This is why the trajectory is composed of lateral and diagonal segments. Two different marks in the plot, i.e., + and $\times$, denote the states where the measurement of either $F_{Y}$ or $M_{X}$ went through zero during an operation. The aggregate of each type of marks corresponds to the line of intersection of the two plains of (3) and $F=0$, or that of (4) and $M=0$.

Based on the analysis of the work trajectories like those in Fig. 6, the most basic strategy when searching for accurate operation positions has been determined as described below:

(I) Measure the condition of $M$ when $F=0$.

(II) Rotate the hand in such a direction as to reduce the measurement of $M$ to some extent.

(III) Translate the hand in such a direction as to make $F=0$ again.

(IV) Examine whether the measurement of $M$ has become smaller than that was measured in (I).

(V) Go back to (I) if the condition of $M$ is out of the allowable range, and then continue applying the same pair of operations if $M$ is decreased by them (otherwise they should be modified).

When taking the functions to move the force and moment pointers in the FID into consideration, the robot operations can be divided into two classes: One is the class of operations for bringing the two pointers together, and the other is the one for moving them toward the origin. In the strategy mentioned above, the former function is assigned to the rotation of the hand while the latter function is assigned to the translation of the hand. The mapping will vary according to the mechanistic factors, such as the length of the beam structure and the position of the center of rotation. Therefore, the operators have to figure out which of translations or rotations are suitable for each function depending on each work condition.

Fig. 7 illustrates this basic operation strategy using a pattern diagram in which each strategic step is labeled with a corresponding number. This visualization helps us understand the existence of an invariant reference frame,

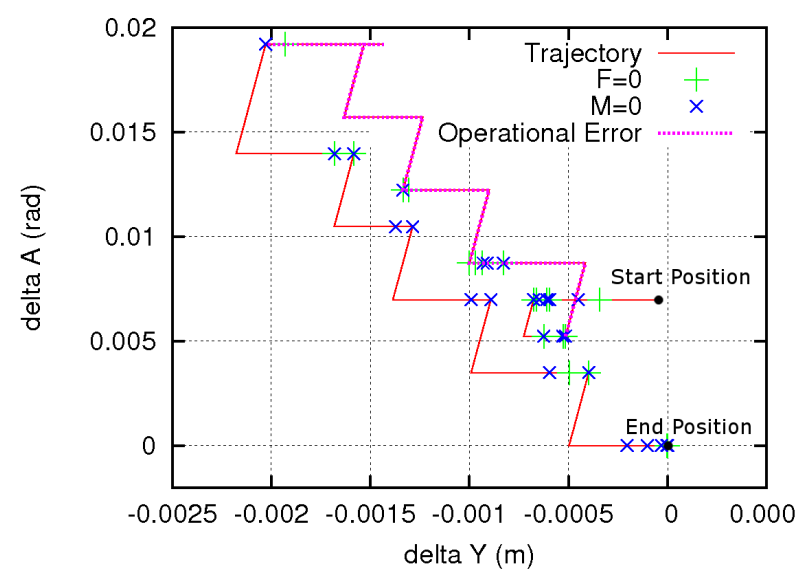

Fig. 8. State trajectory including erroneous operations

which provides a useful basis for evaluating the operations applied thus far. The $F=0$ line forms a frame of reference in this case while the $M=0$ and $F=M / l$ lines can also play a role in the reference frame. Step (I) provides not only information about the current work state but also a reference point for subsequent operations. Implementing (II) and (III) moves the system to the next reference point, and the set of reference points enables the human operator to confirm the adequacy of his decision in (IV).

On the other hand, Fig. 8 shows another teaching work instance, but one that includes some erroneous operations. During an initial phase of this work, the operator selected an adequate pair of operations, but he then made mistakes in selecting the search direction. The duration emphasized by the "Operational Error" line corresponds to those missteps, which is followed by the recovered path to the goal.

Several conceivable factors can be considered for why the operators would make wrong choices. For example, the cases mentioned in the previous paragraph were observed when the iterated operations for (II) and (III) intervened with other types of operations such as opening or closing of the hand, the translation or rotation in another longitudinal plane, and so on. An operator could easily get confused by such interruptions. In order for position teaching to steadily progress, some information resources that provide memory aids and such decision support should be distributed in the work environment.

\section{USER INTERFACE DESIGN}

We have developed a prototype GUI that can provide cognitive support for the whole activity in search of an accurate robot position after the analysis conducted so far. The GUI is a configural display that represents the activity-related information in a way that allows the operators to extract not only the information concerning individual measurements but also higher-order information, which encourages their strategic search operations.

\subsection{Information Requirements}

The analytical results of the work domain and of the operation strategy of position teaching point out that, in 


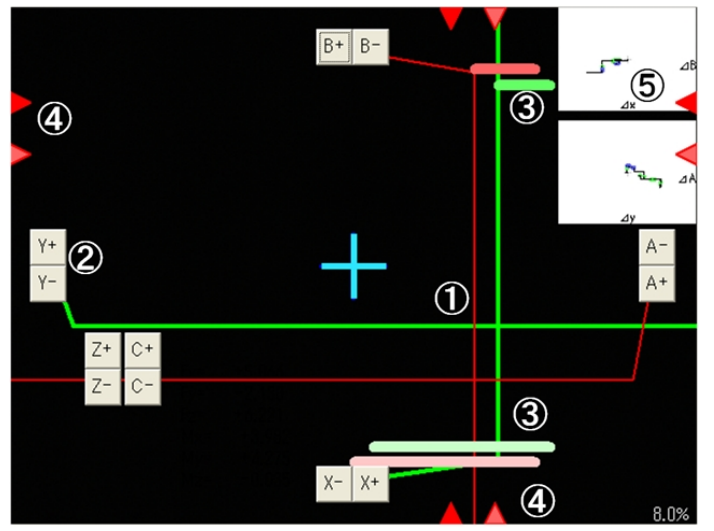

Fig. 9. Proposed GUI

addition to the force and moment visualization, the following items are requirements needing to be externalized in order to assist operators to consistently making decisions:

(A) the history of the operator's inputs for assuring a certain pair of operations are repeated even when other kinds of operations intervene with it,

(B) the latest reference point used to evaluate the current state of the tool-workpiece system, and

(C) the current set of reference points for understanding the force-displacement relationship inherent in the work system.

From (A) to (C), the level of decision-making on the robot operation increases, and thus, the time span increases. These were taken into account when considering our new GUI design for position teaching.

\subsection{Composition of GUI}

Fig. 9 shows the GUI we have developed. For the purpose of illustration, the background of the screen is filled with black in this figure, but normally a live camera image is displayed that captures the area around the robot hand in the same way as in Fig. 4.

The GUI is composed of the following components.

(1) The force and moment measurements taken by the force/torque sensor: Two different cross lines represent $\left(F_{X}, F_{Y}\right)$ and $\left(M_{Y} / l, M_{X} / l\right)$. The former is illustrated using the green lines while the latter the red ones. Both of them must move into the cross hairs at the center of the screen.

(2) The operation buttons used to manipulate the position and orientation of the robot hand: Their locations on the screen correspond to the display axes for the force and moment measurements.

(3) The bars representing the changes in the amount of force and moment during the repetition of a same pair of operations: The $\mathrm{X}$-axis and B-axis operations, and the $\mathrm{Y}$-axis and $\mathrm{A}$-axis operations constitute respective pairs. As long as the same pair is applied to the system, the bars associated with them will grow by the operations. When either operation of the other pair has been selected, they disappear from the screen. The bars on the side of the last operation are filled with deeper colors than the ones before.
(4) The pointers to notate the overlapping positions of the cross lines of (1): Two wedges on each side of the screen indicate the last two positions at which the $F_{X}$ and $M_{Y} / l$ lines, or the $F_{Y}$ and $M_{X} / l$ lines has overlapped. In the same way for the (3) bars, the last overlapping positions are a deeper red than the ones before.

(5) The work trajectories and markers for reference: Two trajectory displays equivalent to Fig. 6 are shown, one of which is on the X-B surface and the other of which is on the $\mathrm{Y}-\mathrm{A}$ surface. For the trajectories on the $\mathrm{X}-\mathrm{B}$ surface, the points that satisfy the $F_{X}=0$ or $F_{X}=M_{Y} / l$ conditions are highlighted by specific markers while for the trajectories on the Y-A surface the $F_{Y}=0$ or $F_{Y}=M_{X} / l$ points are highlighted.

The amount of information held in the (1) component equals to that shown in Fig. 4, whereas the (2) layout affords an operator's "direct manipulation" to some extent (not completely). The remaining three components add significant value to the GUI. The (3), (4), and (5) components are designed for the $(\mathrm{A}),(\mathrm{B})$, and $(\mathrm{C})$ information requirements, respectively. They are the distributed resources that can provide information to support different granularities of an activity in position teaching.

\section{CONCLUSION}

A confined work domain for robot teaching was analyzed using a mechanical explanation model to capture the forcedisplacement relationship inherent in the work system. The resulting model was used to analyze the robot operators' decisions in search of accurate operation positions with the aid of force and moment information, and we were able to clarify a rational operation strategy to (implicitly) make use of an invariant frame of reference in the position search space, such as the virtual line at $F=0$. Based on these findings, a prototype GUI was developed that can provide effective information support for different granularities of activity in position teaching. The GUI is expected to encourage teaching worker's intuitive and strategic robot operations, and it is going to be tested in a future experiment.

\section{REFERENCES}

Burns, C.M. and Hajdukiewicz, J.R. (2004). Ecological Interface Design. CRC Press.

Mitsubishi Electric Corporation (2009). Mitsubishi Industrial Robot INSTRUCTION MANUAL: Controller setup, basic operation, and maintenance.

Rasmussen, J. (1986). Information Processing and Human-Machine Interaction: An Approach To Cognitive Engineering. North-Holland.

Shepherd, A. (2000). Hierarchical Task Analysis. Taylor \& Francis.

Vicente, K.J. (1999). Cognitive Work Analysis: Toward Safe, Productive, and Healthey Computer-Based Work. Lawrence Erlbaum Assoc Inc.

Vicente, K.J. (2002). Ecological interface design: Progress and challenges. Human Factors, 44, 62-78.

Vicente, K.J. and Rasmussen, J. (1990). The ecology of human-machine systems II: Mediating "direct perception" in complex work domain. Ecological Psychology, $2(3), 207-249$. 